\title{
Role of peptidylarginine deiminase type 4 in gastric cancer
}

\author{
JIANG XIN ${ }^{1,2}$ and XIUQI SONG ${ }^{1,3}$ \\ ${ }^{1}$ Qingdao University Medical College, Qingdao, Shandong 266003;
${ }^{2}$ Department of General Surgery, The Third People's Hospital of Qingdao, Qingdao, Shandong 266004;
${ }^{3}$ Department of General Surgery, Qingdao Central Hospital, Qingdao, Shandong 266402, P.R. China
}

Received July 9, 2015; Accepted August 1, 2016

DOI: $10.3892 /$ etm.2016.3798

\begin{abstract}
Peptidylarginine deiminase type 4 (PADI4) post-translationally converts peptidylarginine to citrulline, appearing to be overexpressed in numerous carcinomas. The current study aimed to investigate the expression of PADI4 in gastric cancer tissues and its effect on the biological activities of SGC-7901 and AGS tumor cell lines. The expression of PADI4 was determined in gastric cancer and normal gastric mucosa tissues using western blot analysis and reverse transcription-quantitative polymerase chain reaction. Gastric cancer cell lines were divided into the following groups: Mock group (subjected to transfection reagent); negative group [subjected to small interfering RNA (siRNA) transfection]; PADI4 siRNA group (subjected to PADI4 siRNA transfection); 5-fluorouracil (5-Fu) group (subjected to 5-Fu); and 5-Fu + siRNA transfection group (subjected to 5-Fu and PADI4 siRNA transfection). The effects of silencing PADI4 with the above measures on the proliferation and invasion of SGC-7901 and AGS cells were determined by MTT and Transwell chamber assays. In addition, propidium iodide staining was performed to detect the effects of PADI4 on the cell cycle. A significant increase in the expression of PADI4 mRNA in gastric cancer tissue compared with normal mucosa tissue was identified $(\mathrm{P}<0.05)$. The proliferation and invasion of SGC-7901 and AGS cells were significantly decreased in the PADI4 siRNA group. Furthermore, flow cytometry DNA analysis revealed that silencing PADI4 resulted in significant $\mathrm{S}$ phase arrest and marked decrease of cells in the G2/M phase. PADI4 siRNA coupled with 5-Fu significantly enhanced its inhibitory effect on the proliferation of gastric cancer cells. In conclusion, PADI4 demonstrated high expression in gastric cancer and served an important role in the biological activities of gastric cancer cells involving cell proliferation, invasion and
\end{abstract}

Correspondence to: Dr Xiuqi Song, Department of General Surgery, Qingdao Central Hospital, 127 Siliunan Road, Qingdao, Shandong 266402, P.R. China

E-mail: songxq0187@yeah.net

Key words: peptidylarginine deiminase 4, gastric cancer, gene silencing, cell cycle, cell proliferation cell cycle. As a result, PADI4 may be a valid cancer susceptibility gene and potential target for cancer therapy.

\section{Introduction}

The overall incidence of gastric cancer has decreased in a number of countries as a result of extensive diagnostic and therapeutic investigations. However, gastric cancer remains a major global health burden and is the second most common cause of cancer-related mortality (1). The geographic distribution of gastric cancer is characterized by wide international variations, particularly in high-risk areas such as Japan, Korea, China, and South and Central America (2).

To date, molecular and genetic abnormalities are responsible for the development and progression of gastric cancer, including the activation of oncogenes and inactivation of various tumor suppressor genes $(3,4)$. As a result of gene alteration, the downstream signal transduction pathways will be consequently affected, involving the control of diverse cellular functions such as cell growth, differentiation, metastasis and adhesion $(5,6)$. Wang et al (7) indicated that specificity protein 1 (Spl) was significantly increased in gastric tumor specimens and associated with patient survival, suggesting that abnormal Sp1 expression contributes towards the development and progression of gastric cancer. Kurayoshi et al (8) proposed that the overexpression of Wnt family member 5A improved the migration and invasion ability of gastric cancer cells and was associated with the aggressiveness and poor prognosis of gastric cancer. Further evidence demonstrated that the restoration of Kruppel-like factor 4 expression resulted in the marked suppression of gastric cancer cell growth in vitro and significant attenuation of tumor growth in an animal model, suggesting that it may serve as a prognostic marker and potential therapeutic target for gastric cancer (9).

Peptidylarginine deiminase type 4 (PADI4), one of four known PADI genes, is characterized by the conversion of peptidylarginine into citrulline in the process of citrullination. The gene is expressed in hematopoietic progenitor cells, granulocytes, T cells, B cells, macrophages and natural killer cells (10). Accumulating evidence suggests that PADI4 appears to be overexpressed in numerous types of carcinomas, including breast carcinoma, hepatocarcinoma, renal, bladder and lung carcinoma (11). Chang et al (12) indicated that the expression levels of PADI4 mRNA and protein are significantly enhanced in breast fibroadenoma and thyroid 
adenoma compared with surrounding healthy tissues. In addition, one study suggested that PADI4 was expressed at higher levels in ovarian adenocarcinoma, and confirmed this using reverse transcription-quantitative polymerase chain reaction (RT-qPCR) and western blot analyses (13). Ordóñez et al (14) suggested that PADI4 may have an effect on tumor progression with regards to the elevation of citrullinated antithrombin levels in serum samples of colorectal adenocarcinoma patients. On this basis, the detection of genetic abnormalities of PADI4 may provide an alternative opportunity for the early diagnosis and clinical interventions of various cancers.

In the present study, the expression of PADI4 was detected in gastric cancer and normal gastric mucosa tissues. PADI4 was suppressed with the aid of small interfering RNA (siRNA) and 5-fluorouracil (5-Fu), and the effects of silencing PADI4 on various cell functions of SGC-7901 and AGS cells were determined in order to explore the pathogenic role of PADI4 in gastric cancer.

\section{Materials and methods}

Materials and reagents. A total of 10 tissues samples from patients with gastric cancer (6 men and 4 women) between February 2010 and October 2012 were obtained at the Third People's Hospital of Qingdao (Qingdao, China). Ten normal gastric mucosa tissues (a distance away from the resected margin of gastric cancer) were obtained from these patients to use as a control. All samples were confirmed by pathological analysis and were not treated with radiotherapy and chemotherapy prior to surgery. The median age in the cohort of patients was 55.3 \pm 5.9 years (range, 47.6-57.6 years). All samples were stored at $-80^{\circ} \mathrm{C}$ for further analysis. Written informed consent was obtained from all the patients. The study was conducted with approval from the Ethics Committee of the Third People's Hospital of Qingdao.

Roswell Park Memorial Institute (RPMI)-160 culture medium, fetal bovine serum (FBS) and double antibody (penicillin-streptomycin) were obtained from Gibco (Thermo Fisher Scientific, Inc., Waltham, MA, USA). PADI4 and GAPDH sheep anti-rabbit antibodies, and secondary antibody, were obtained from Santa Cruz Biotechnology, Inc. (Santa Cruz, CA, USA). Radioimmunoprecipitation assay (RIPA) lysis buffer, polyvinylidene difluoride (PVDF) membrane and Bradford Protein Quantitative kit were purchased from Beyotime Institute of Biotechnology (Jiangsu, China). Enhanced Chemiluminescence (ECL) kit was obtained from Invitrogen (Thermo Fisher Scientific, Inc.). Bovine serum albumin (BSA) was obtained from Sangon Biotechnology Co., Ltd. (Shanghai, China). Total RNA Extraction kit was obtained from Omega Bio-tek, Inc. (Doraville, GA, USA). Quantitative PCR kit and SYBR Green qPCR Master Mix was obtained from Hoffmann-La Roche, Inc. (Basel, Switzerland). 5-Fu was obtained from Anji Haosen Pharmaceutical Co., Ltd. (Jiangsu, China).

Cell culture and treatment with siRNA and 5-Fu. Human gastric cancer cell lines (SGC-7901 and AGS) were cultured in RPMI-1640 supplemented with $10 \% \mathrm{FBS}$ at $37^{\circ} \mathrm{C}$ with $5 \% \mathrm{CO}_{2}$ in a humidified atmosphere. Cells in logarithmic growth phase were resuspended and seeded in a 6-well plate at a density of $5 \times 10^{5}$ cells $/ \mathrm{ml}$. Subsequently, transient transfection was performed when cells reached $80 \%$ confluence. For the experimental transfection, samples were divided into the following groups: Mock group (subjected to transfection reagent); negative group (subjected to siRNA transfection); PADI4 siRNA group (subjected to PADI4 siRNA transfection); 5-Fu group (subjected to 5-Fu); and 5-Fu+ ${ }^{+}$siRNA transfection group (treated with 5-Fu and subjected to PADI4 siRNA transfection). All transfection procedures were performed according to the manufacturer's instructions of Lipofectamine 2000. The interference sequences in the PADI4 siRNA and negative group were as follows: siPADI4 sense, 5'GAAGGAGUUUCC CAUCAAATT'3 and antisense, 5'UUUGAUGGGAAACUC CUUCAG'3; negative control sense, UUCUCCGAACGUGUC ACG and antisense, 5'ACGUGACACGUUCGGAGAATT'3.

Western blot analysis. Fresh tissues were cut into sections and lysed in RIPA buffer on ice. Cell lysates were centrifuged at $12,000 \mathrm{rpm}$ for $10 \mathrm{~min}$, and the protein supernatant was transferred into new tubes. Subsequently, the protein concentration of each sample was determined using BCA Protein Assay kit (Pierce; Thermo Fisher Scientific, Inc.). For SDS-PAGE, $20 \mu \mathrm{g}$ of the total protein from each sample was resolved using $12 \%$ SDS-PAGE and transferred to PVDF membranes for $45 \mathrm{~min}$ using a tank transfer system (Bio-Rad Laboratories, Inc., Hercules, CA, USA). Then, the membranes were blocked in Tris-buffered saline containing Tween 20 (TBST) with 3\% $\mathrm{BSA}$ at $37^{\circ} \mathrm{C}$ for $1 \mathrm{~h}$, and incubated with PADI4 primary antibody (1:500; cat. no. sc-365369) overnight at $4^{\circ} \mathrm{C}$. After washing three times with TBST, the membranes were incubated with anti-rabbit secondary antibody (1:500; cat. no. sc-98991) for $1 \mathrm{~h}$ at room temperature. Proteins were detected using an ECL kit according to the manufacturer's instructions. GAPDH was used as an internal control.

$R T-q P C R$. Total RNA from the gastric tissues was extracted with TRIzol reagent according to the manufacturer's instructions as previously described (15). cDNA synthesis was performed with $500 \mathrm{ng}$ RNA using the Superscript RT kit (Invitrogen; Thermo Fisher Scientific, Inc.). RT-qPCR amplification was performed using the Mx3000P QPCR System (Agilent Technologies, Inc., Santa Clara, CA, USA) with the primers listed in Table I. As an internal control for qPCR, GAPDH mRNA expression was amplified from the same cDNA samples. All results were normalized to GAPDH amplification. PCR reactions were performed in a total volume of $20 \mu \mathrm{l}$, containing $15 \mu \mathrm{l} 1 \mathrm{X}$ SYBR Green Supermix, $1 \mu 1$ each specific primer, $2 \mu \mathrm{l}$ water and $1 \mu \mathrm{l} \mathrm{cDNA}$ template. The amplification reaction conditions were as follows: Pre-denaturation at $90^{\circ} \mathrm{C}$ for $3 \mathrm{~min}$; followed by 40 cycles of denaturation at $94^{\circ} \mathrm{C}$ for $30 \mathrm{sec}$; annealing at $30^{\circ} \mathrm{C}$ for $30 \mathrm{sec}$; and extension at $72^{\circ} \mathrm{C}$ for $40 \mathrm{sec}$. The $2^{-\Delta \Delta \mathrm{Cq}}$ method was applied to analyze the relative changes in gene expression from RT-qPCR experiments, as previously described (16).

MTT assay. An MTT assay was performed to determine the effect of PADI4 on the cellular growth of SGC-7901 and AGS cells, as previously described (17). Briefly, SGC-7901 and AGS cells at a density of $5 \times 10^{3}$ cells $/ \mathrm{ml}$ were seeded in 96-well plates and cultured with the various transfections or $5-\mathrm{Fu}$, as 
Table I. Specific primers of PADI4, MMP2, MMP9 and GAPDH.

\begin{tabular}{lcc}
\hline Gene & Forward primer & Reverse primer \\
\hline PADI4 & 5'-GGGGACATTGATCCGTGTGA-3' & 5'-TCGTC AGGGTCACCTCTACC-3' \\
MMP2 & 5'-CCAGCTGGCCTAGTGATGAT-3' & 5'-CCGCATGGTCTCGATGGTAT-3' \\
MMP9 & 5'-TCTATGGTCCTCGCCCTGAA-3' & 5'-CATCGTCCACCGGACTCAAA-3' \\
GAPDH & 5'-AATGGGCAGCCGTTAGGAAA-3' & 5'-GCGCCCAATACGACCAAATC-3'
\end{tabular}

PADI4, peptidylarginine deiminase type 4; MMP, matrix metalloproteinase.

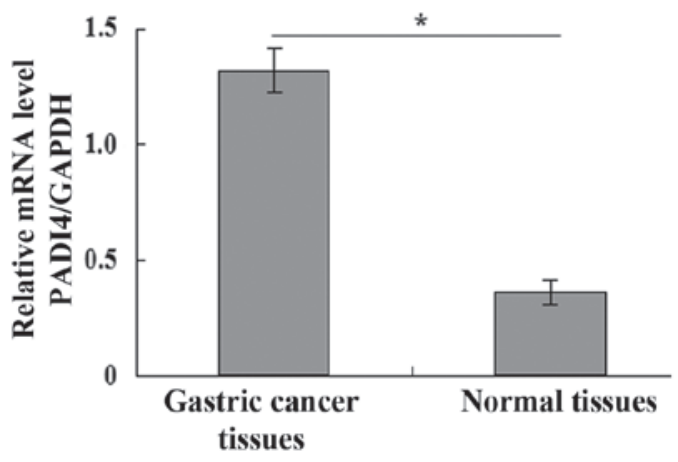

Figure 1. Expression of PADI4 in gastric cancer and normal mucosa tissue. ${ }^{*} \mathrm{P}<0.05$. PADI4, peptidylarginine deiminase type 4 .

described above, for 24, 48 and $72 \mathrm{~h}$. Subsequently, $20 \mu \mathrm{l}$ of MTT (5 mg/ml; Gefan Biotech, Shanghai China) was added to each well and incubated for $4 \mathrm{~h}$ at $37^{\circ} \mathrm{C}$. Then, $150 \mu \mathrm{l}$ dimethyl sulfoxide was added to each well, and the mixture was shaken in a horizontal direction for $10 \mathrm{~min}$ to dissolve the produced formazan crystals. The optical density (OD) value at $490 \mathrm{~nm}$ of each sample was measured using a plate reader.

Flow cytometry assay. Cell cycle was determined by flow cytometry, as previously described (18). Briefly, cells in the logarithmic growth phase were collected subsequent to transfection with PADI4 siRNA for $48 \mathrm{~h}$. Cells were fixed with $70 \%$ ethanol for $24 \mathrm{~h}$ followed by washing in PBS three times. Subsequently, cells were treated with $50 \mu$ l RNAse solution $(0.5 \mathrm{mg} / \mathrm{ml})$ at $37^{\circ} \mathrm{C}$ for $30 \mathrm{~min}$. Then, cells were incubated with propidium iodide $(50 \mu \mathrm{g} / \mathrm{ml})$ for staining at $4^{\circ} \mathrm{C}$ for 30 min under dark conditions and filtered with a stainless steel screen (200 mesh). The cell cycle of each sample was detected using flow cytometry. Experiments were repeated three times.

Cell invasion assay. Cell invasion was identified using a Transwell cell culture chamber $(8 \mu \mathrm{m}$; Corning Incorporated, Corning, NY, USA), as previously described (19). Briefly, SGC-7901 and AGS cells were suspended in serum-free RPMI-1640 culture medium at a concentration of $1 \times 10^{5}$ cells $/ \mathrm{ml}$ following transfections, as described above. Cell suspension $(200 \mu \mathrm{l})$ was then added to the upper chamber $\left(2 \times 10^{4}\right.$ cells/well). Simultaneously, RPMI-1640 media supplemented with $20 \%$ FBS was added to the lower compartment. Then, the Transwell chamber was incubated at $37^{\circ} \mathrm{C}$ in a $5 \% \mathrm{CO}_{2}$ atmosphere for $24 \mathrm{~h}$. Subsequently, cells in the upper compartment were collected and fixed with formaldehyde solution. Following staining with hematoxylin and eosin, five fields of each image were randomly selected at higher magnification using a confocal microscope, and the number of cells were calculated. Each assay was repeated three times.

Statistical analysis. Statistical analyses were performed by one-way analysis of variance using SPSS version 13.0 (SPSS, Inc., Chicago, IL, USA). Data are presented as mean \pm standard deviation. Student's t-test was performed to evaluate inter-group comparisons. $\mathrm{P}<0.05$ was considered to indicate a statistically significant difference.

\section{Results}

Expression of PADI4 in gastric cancer and normal mucosa tissue. Fig. 1 presents the expression levels of PADI4 in gastric cancer and normal mucosa tissues. A significant increase in PADI4 protein mRNA expression levels were identified in gastric cancer tissue compared with normal mucosa tissue $(\mathrm{P}<0.05$; Fig. 1). Furthermore, the expression of PADI4 $(1.367 \pm 0.268)$ in gastric cancer tissue was significantly higher compared with normal mucosa tissue $(0.429 \pm 0.335)$, analyzed by RT-qPCR analysis $(\mathrm{P}<0.05)$.

Effects of silencing PADI4 on the proliferation, apoptosis and invasion of SGC-7901 and AGS cells. Following silencing PADI4 with siRNA, the expression level of PADI4 was markedly decreased compared with the mock and negative group (Fig. 2A). A significant decrease $(\mathrm{P}<0.05)$ in the proliferation of SGC-7901 and AGS cells in the PADI4 siRNA group was identified in comparison with the mock and negative groups at 36 and 48 h (Fig. 2B). In addition, marked S phase arrest and a marked decrease in the number of gastric cancer cells in the G2/M phase was identified in the PADI4 siRNA group (Fig. 3). Furthermore, the invasion of SGC-7901 and AGS cells were markedly decreased when treated with PADI4 siRNA (Fig. 4).

Effect of silencing PADI4 on the expression of matrix metalloproteinase (MMP) 2 and MMP9 in gastric cancer cells. After silencing PADI4 in SGC-7901 cells, MMP2 and MMP9 expression levels were lower compared with the mock and negative control groups (Fig. 2A).

Synergistic effect of PADI4 siRNA and 5-Fu on the proliferation of SGC-7901 and AGS cells. A marked decrease was observed in the proliferation of gastric cancer cells in the PADI4 siRNA, 5-Fu and PADI4 siRNA + 5-Fu groups 


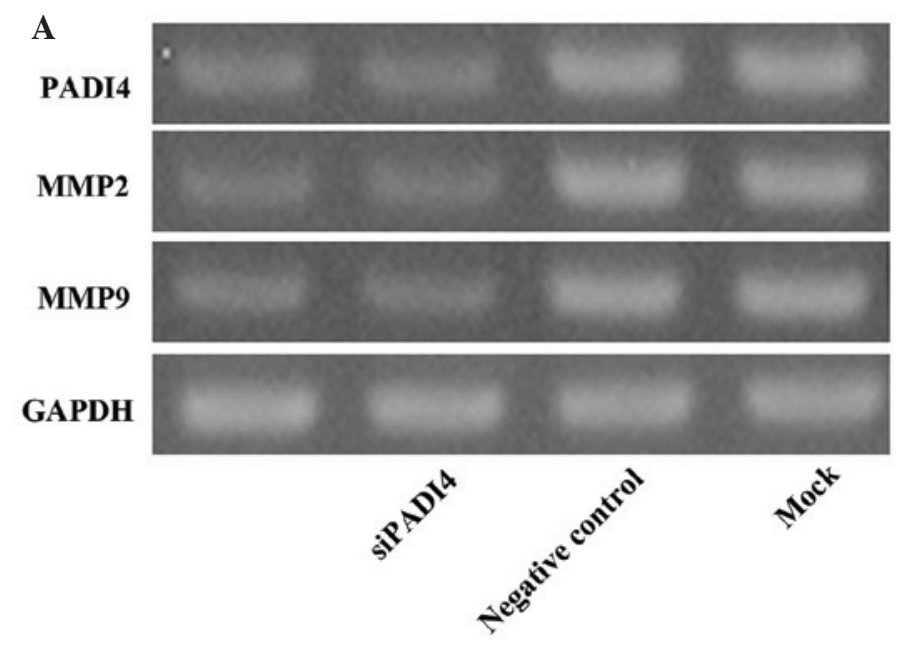

B
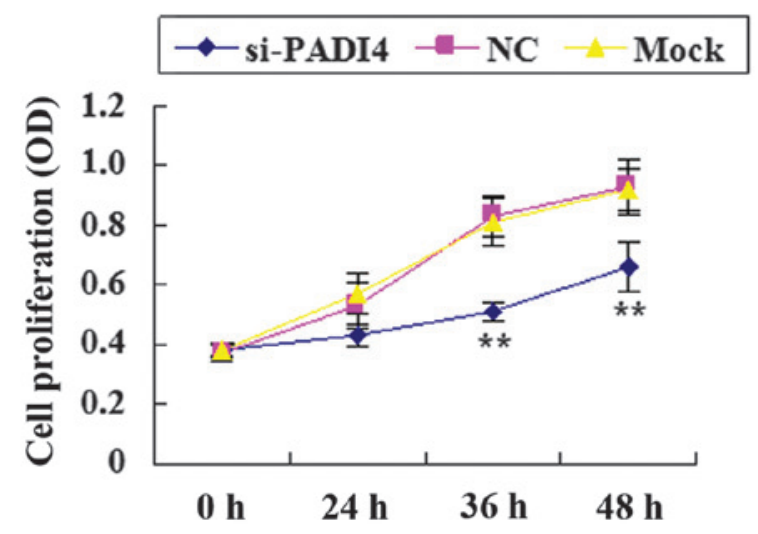

Figure 2. (A) Expression of PADI4, MMP2 and MMP9 was analyzed in SGC-7901 cells. (B) The effect of silencing PADI4 on cell proliferation was measured by MTT assay. ${ }^{* *} \mathrm{P}<0.05$ vs. the Mock and NC groups. PADI4, peptidylarginine deiminase type 4; si-PADI4, small interfering-PADI4; NC, negative control; OD, optical density; MMP, matrix metalloproteinase.

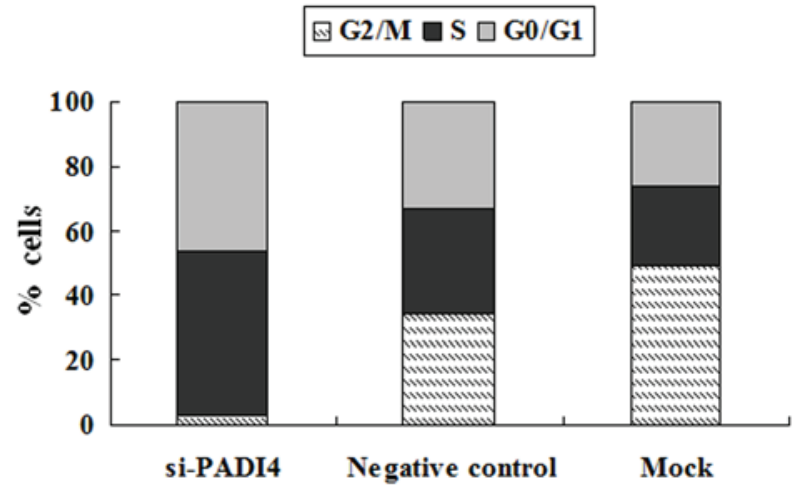

Figure 3. Influence of silencing PADI4 on the cell cycle in si-PADI4, NC and mock gastric cancer cells. PADI4, peptidylarginine deiminase type 4; si-PADI4, small interfering-PADI4; NC, negative control.

compared with the mock and control groups. The inhibitory effect was most prominent when PADI4 siRNA and 5-Fu were combined. Furthermore, a significant difference $(\mathrm{P}<0.05)$ was identified in the combination group at $36 \mathrm{~h}$ compared with the PADI4 siRNA group and the 5-Fu group (Fig. 5).

\section{Discussion}

PADI4 is one member of the PADI gene family catalyzing protein cirtullination in the presence of $\mathrm{Ca}^{2+}$. A number of reports have suggested that citrullination is associated with specific biological events involving apoptosis, inflammation, histone-related gene expression and trauma (20-22). Extensive studies have focused on the role of PADI4 in rheumatoid arthritis (RA). Evidence indicates that PADI4 presents a strong association with RA by whole genome single nucleotide polymorphism scanning (23). Chang et al (24) demonstrated an increased expression of PADI4 in the synovial fluid and synovial membrane of patients with RA. Iwamoto et al (25) indicated that PADI was positively correlated with RA in Japanese and Caucasian populations of European descent with a meta-analysis. In addition, the role of PADI4 has attracted increasing attention with regards to a number of malignant tumors. Lv et al (26) observed a significant elevation of PADI4 in hepatocellular carcinomas compared with surrounding healthy tissue using western blot analysis. Ulivi et al (27) demonstrated that non-small-cell lung cancer (small-cell lung cancer) could be accurately discriminated according to the expression of PADI4 and pro-platelet basic protein by free circulating DNA analysis.

In the present study, a significant increase in PADI4 mRNA expression levels was identified in gastric cancer tissues compared with normal gastric mucosa tissues, suggesting that there is a correlation between PADI4 and gastric cancer, and that it may serve a role clinical diagnosis. In addition, it was observed that the proliferation and invasion of SGC-7901 and AGS cells were significantly decreased when PADI4 was silenced with siRNA. Furthermore, silencing PADI4 resulted in significant $\mathrm{S}$ phase arrest and a marked decrease in the number of cells in the G2/M phase. Together, these results suggest that overexpression of PADI4 contributes towards gastric cancer cell growth and migration, and that the silencing of PADI4 may provide an alternative treatment for gastric cancer.

MMPs are a family of enzymes with proteolytic activity for degrading various components of the extracellular matrix (ECM). Among these are MMP2 and MMP9 (gelatinase subgroup of MMPs) which serve an important role in the regulation of key signaling pathways in cell invasion, growth, angiogenesis and inflammation, by cleaving numerous different targets, such as cytokines, chemokines, growth factors and ECM (28). Currently, growing research focuses on MMP2 and MMP9 since they are overexpressed in various malignant tumors, and their expression and activity are frequently related to tumor progression and poor prognosis (29). Previous evidence suggests that the expression of MMP2 and MMP9 increases in a numbers of carcinomas, such as brain, ovarian, breast and lung cancers $(30,31)$. Wong et al (32) proposed that MMP2 and MMP9 contribute towards the aggressiveness of highly metastatic forms of nasopharyngeal carcinoma. Matsumura et al (33) indicated that a MMP-9 polymorphism was associated with the depth of tumor invasion and 

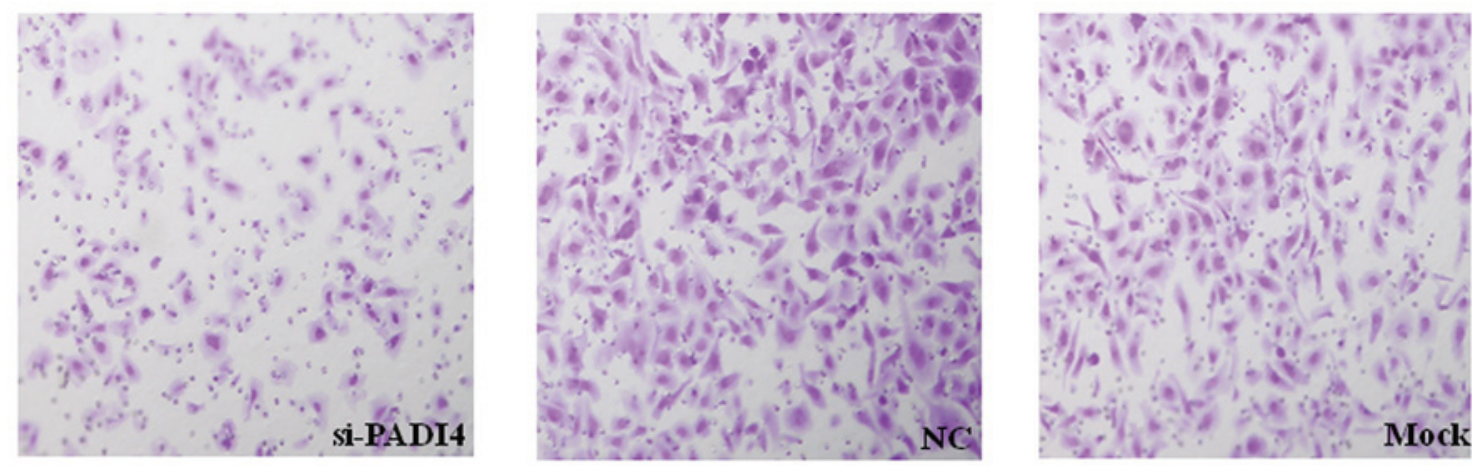

Figure 4. Effects of silencing PADI4 on the invasion of SGC-7901 cells.

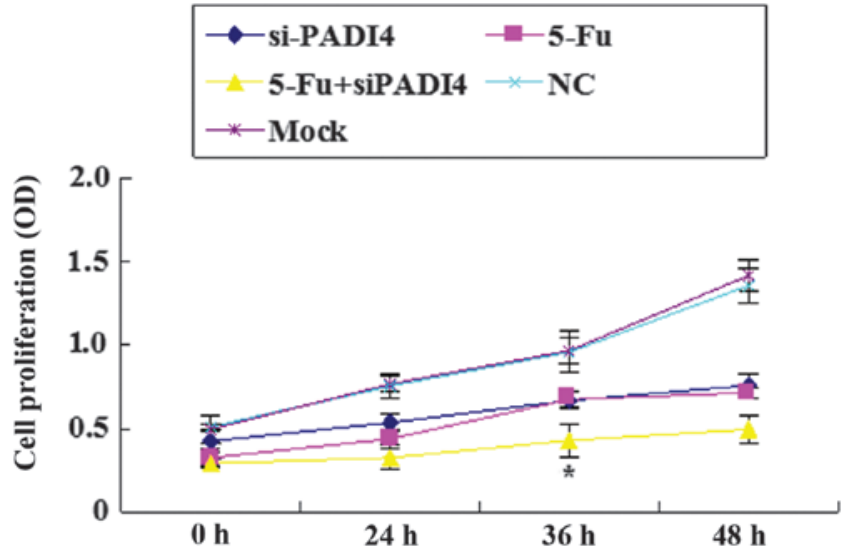

Figure 5. Synergistic effect of PADI4 siRNA and 5-Fu on the proliferation of SGC-7901 and AGS cells. ${ }^{*} \mathrm{P}<0.05$ vs. the PADI4 siRNA and $5-\mathrm{Fu}$ group. PADI4, peptidylarginine deiminase type 4 ; si-PADI4, small interfering-PADI4; NC, negative control; 5-FU, 5-fluorouracil.

tumor, nodes and metastasis classification of gastric cancer. In the current study, the expression of MMP2 and MMP9 was decreased after silencing PADI4 in SGC-7901 and AGS cells, which suggests that PADI4 may contribute towards the abnormal invasion of gastric cancer cells by regulating the expression of MMP2 and MMP9.

Aside from the role of PADI4 in cell invasion, PADI4 serves a crucial role in mediating cell cycle and apoptosis, both of which are associated with the expression of the tumor suppressor p53. Liu et al (34) demonstrated that overexpression of PADI4 could upregulate the expression of p53 and its downstream factors p21 and B-cell lymphoma 2-associated $\mathrm{X}$ protein, result in cell cycle arrest in the G1 phase, and cause mitochondria-mediated apoptosis of human leukemia HL-60 cells and human acute T leukemia Jurkat cells. Further evidence indicated that the depletion of PADI4 by small hairpin RNA increased the population of G1 cells, decreased the population of $\mathrm{S}$ and $\mathrm{G} 2 / \mathrm{M}$ cells and increased cell apoptosis in HCT116 cells in a p53-dependent manner (35). Similar results were obtained in another study, where Yao et al (36) demonstrated that knockdown of PADI4 with $\mathrm{Cl}$-amidine and siRNA decreased cell viability and induced apoptosis of breast cancer MCF-7 cells. In the present study, it was observed that the silencing of PADI4 resulted in an $\mathrm{S}$ cell cycle block with a concomitant decrease of cells in the G2/M phase, and decreased cell growth and invasion. Furthermore, the inhibitory effect of PADI4 siRNA on the proliferation of gastric cancer cells were enhanced when combined with 5-Fu. Together, the results suggest that PAD4 siRNA treatment coupled with 5-Fu may contribute towards the decrease in drug resistance of cancer cells, improve chemotherapeutic effects and provide a possible therapeutic option for treating gastric cancer.

In conclusion, the present study demonstrates the increased expression of PADI4 in gastric cancer tissues. The silencing of PADI4 suppressed the proliferation and invasion of cancer cells, and arrested cell cycle in the $\mathrm{S}$ phase. In addition, the combination of siRNA PADI4 with 5-Fu presented a stronger inhibitory effect on gastric cell growth compared with siRNA PADI4 alone. As a result, PADI4 may be considered as a prognostic indicator of gastric tumor and a potential target for gastric cancer therapy.

\section{References}

1. Roder DM: The epidemiology of gastric cancer. Gastric Cancer 5 (Suppl 1): 5-11, 2002.

2. Parkin DM, Bray FI and Devesa SS: Cancer burden in the year 2000. The global picture. Eur J Cancer 37 (Suppl 1): S4-S66, 2001.

3. Tahara E: Molecular aspects of invasion and metastasis of stomach cancer. Verh Dtsch Ges Pathol 84: 43-49, 2000.

4. Sud R, Wells D, Talbot IC and Delhanty JD: Genetic alterations in gastric cancers from British patients. Cancer Genet Cytogenet 126: 111-119, 2001.

5. Fiocca R, Luinetti O, Villani L, Mastracci L, Quilici P, Grillo F and Ranzani GN: Molecular mechanisms involved in the pathogenesis of gastric carcinoma: Interactions between genetic alterations, cellular phenotype and cancer histotype. Hepatogastroenterology 48: 1523-1530, 2001.

6. El-Rifai W and Powell SM: Molecular biology of gastric cancer. Semin Radiat Oncol 12: 128-140, 2002.

7. Wang L, Wei D, Huang S, Peng Z, Le X, Wu TT, Yao J, Ajani J and Xie K: Transcription factor Sp1 expression is a significant predictor of survival in human gastric cancer. Clin Cancer Res 9: 6371-6380, 2003.

8. Kurayoshi M, Oue N, Yamamoto H, Kishida M, Inoue A, Asahara T, Yasui W and Kikuchi A: Expression of Wnt-5a is correlated with aggressiveness of gastric cancer by stimulating cell migration and invasion. Cancer Res 66: 10439-10448, 2006.

9. Wei D, Gong W, Kanai M, Schlunk C, Wang L, Yao JC, Wu TT, Huang S and Xie K: Drastic down-regulation of Krüppel-like factor 4 expression is critical in human gastric cancer development and progression. Cancer Res 65: 2746-2754, 2005.

10. Liu YL, Chiang YH, Liu GY and Hung HC: Functional role of dimerization of human peptidylarginine deiminase 4 (PAD4). PLoS One 6: e21314, 2011.

11. Chang $X$ and Han J: Expression of peptidylarginine deiminase type 4 (PAD4) in various tumors. Mol Carcinog 45: 183-196, 2006. 
12. Chang X, Han J, Pang L, Zhao Y, Yang Y and Shen Z: Increased PADI4 expression in blood and tissues of patients with malignant tumors. BMC Cancer 9: 40, 2009.

13. Wang L, Chang X, Yuan G, Zhao Y and Wang P: Expression of peptidylarginine deiminase type 4 in ovarian tumors. Int J Biol Sciences 6: 454-464, 2010.

14. Ordóñez A, Yélamos J, Pedersen S, Miñano A, Conesa-Zamora P, Kristensen SR, Stender MT, Thorlacius-Ussing O, Martínez-Martínez I, Vicente V and Corral J: Increased levels of citrullinated antithrombin in plasma of patients with rheumatoid arthritis and colorectal adenocarcinoma determined by a newly developed ELISA using a specific monoclonal antibody. Thromb Haemost 104: 1143-1149, 2010.

15. Simms D, Cizdziel PE and Chomczynski P: TRIzol: A new reagent for optimal single-step isolation of RNA. Focus 15: 532-535, 1993.

16. Livak KJ and Schmittgen TD: Analysis of relative gene expression data using real-time quantitative PCR and the $2^{-\Delta \Delta C T}$ method. Methods 25: 402-408, 2001.

17. Tiffen JC, Bailey CG, Ng C, Rasko JE and Holst J: Luciferase expression and bioluminescence does not affect tumor cell growth in vitro or in vivo. Mol Cancer 9: 299, 2010.

18. Haase SB and Reed SI: Improved flow cytometric analysis of the budding yeast cell cycle. Cell Cycle 1: 132-136, 2002.

19. Katz M, Amit I, Citri A, Shay T, Carvalho S, Lavi S, Milanezi F Lyass L, Amariglio N, Jacob-Hirsch J, et al: A reciprocal tensin-3-cten switch mediates EGF-driven mammary cell migration. Nat Cell Biol 9: 961-969, 2007.

20. Wang Y, Wysocka J, Sayegh J, Lee YH, Perlin JR, Leonelli L, Sonbuchner LS, McDonald CH, Cook RG, Dou Y, et al: Human PAD4 regulates histone arginine methylation levels via demethylimination. Science 306: 279-283, 2004.

21. Klose RJ and Zhang Y: Regulation of histone methylation by demethylimination and demethylation. Nat Rev Mol Cell Biol 8 : 307-318, 2007.

22. Cuthbert GL, Daujat S, Snowden AW, Erdjument-Bromage H, Hagiwara T, Yamada M, Schneider R, Gregory PD, Tempst P, Bannister AJ and Kouzarides T: Histone deimination antagonizes arginine methylation. Cell 118: 545-553, 2004.

23. Suzuki A, Yamada R, Chang X, Tokuhiro S, Sawada T, Suzuki M, Nagasaki M, Nakayama-Hamada M, Kawaida R, Ono M, et al: Functional haplotypes of PADI4, encoding citrullinating enzyme peptidylarginine deiminase 4, are associated with rheumatoid arthritis. Nat Genet 34: 395-402, 2003.

24. Chang X, Zhao Y, Sun S, Zhang Y and Zhu Y: The expression of PADI4 in synovium of rheumatoid arthritis. Rheumatol Int 29 1411-1416, 2009.
25. Iwamoto T, Ikari K, Nakamura T, Kuwahara M, Toyama Y, Tomatsu T, Momohara S and Kamatani N: Association between PADI4 and rheumatoid arthritis: A meta-analysis. Rheumatology (Oxford) 45: 804-807, 2006.

26. Lv Y, Xia Y, Wang Y and Cai C: Expression of PADI4 in hepatocellular carcinoma. Chinese-German J Clin Oncol 8: 453-455, 2009.

27. Ulivi P, Mercatali L, Casoni GL, Scarpi E, Bucchi L, Silvestrini R, Sanna S, Monteverde M, Amadori D, Poletti V and Zoli W: Multiple marker detection in peripheral blood for NSCLC diagnosis. PLoS One 8: e57401, 2013.

28. Kessenbrock K, Plaks V and Werb Z: Matrix metalloproteinases: Regulators of the tumor microenvironment. Cell 141: 52-67, 2010.

29. Bauvois B: New facets of matrix metalloproteinases MMP-2 and MMP-9 as cell surface transducers: Outside-in signaling and relationship to tumor progression. Biochim Biophys Acta 1825: 29-36, 2012.

30. Roy R, Yang J and Moses MA: Matrix metalloproteinases as novel biomarkers and potential therapeutic targets in human cancer. J Clin Oncol 27: 5287-5297, 2009.

31. Turpeenniemi-Hujanen T: Gelatinases (MMP-2 and -9) and their natural inhibitors as prognostic indicators in solid cancers. Biochimie 87: 287-297, 2005.

32. Wong TS, Kwong DL, Sham JS, Wei WI, Kwong YL and Yuen AP: Clinicopathologic significance of plasma matrix metalloproteinase-2 and -9 levels in patients with undifferentiated nasopharyngeal carcinoma. Eur J Surg Oncol 30: 560-564, 2004.

33. Matsumura S, Oue N, Nakayama H, Kitadai Y, Yoshida K, Yamaguchi Y, Imai K, Nakachi K, Matsusaki K, Chayama K and Yasui W: A single nucleotide polymorphism in the MMP-9 promoter affects tumor progression and invasive phenotype of gastric cancer. J Cancer Res Clin Oncol 131: 19-25, 2005.

34. Liu GY, Liao YF, Chang WH, Liu CC, Hsieh MC, Hsu PC, Tsay GJ and Hung HC: Overexpression of peptidylarginine deiminase IV features in apoptosis of haematopoietic cells. Apoptosis 11: 183-196, 2006.

35. Li P, Yao H, Zhang Z, Li M, Luo Y, Thompson PR, Gilmour DS and Wang Y: Regulation of p53 target gene expression by peptidylarginine deiminase 4. Mol Cell Biol 28: 4745-4758, 2008.

36. Yao H, Li P, Venters BJ, Zheng S, Thompson PR, Pugh BF and Wang Y: Histone Arg modifications and p53 regulate the expression of OKL 38 , a mediator of apoptosis. J Biol Chem 283: 20060-20068, 2008. 